\title{
Gauge invariance and QCD twist-3 factorization for single spin asymmetries
}

\author{
J.P. Ma ${ }^{a, b}$ and G.P. Zhang ${ }^{b}$ \\ ${ }^{a}$ State Key Laboratory of Theoretical Physics, Institute of Theoretical Physics, Academia Sinica, \\ P.O. Box 2735, Beijing 100190, China \\ ${ }^{b}$ Center for High-Energy Physics, Peking University, \\ Beijing 100871, China \\ E-mail: majp@itp.ac.cn, zgp-phys@pku.edu.cn
}

ABSTRACT: The collinear factorization at twist-3 for Drell-Yan processes is studied with the motivation to solve the discrepancy in literature about the single spin asymmetry in the lepton angular distribution, and to show how QCD gauge invariance is realized in the hadronic tensor. The obtained result here agrees with our early result derived with a totally different approach. In addition to the asymmetry we can construct another two observables to identify the spin effect. We show that the gauge invariance of different contributions in the hadronic tensor is made in different ways by summing the effects of gluon exchanges. More interestingly is that we can show that the virtual correction to one structure function of the hadronic tensor, hence to some weighted SSA observables, is completely determined by the quark form factor. This will simplify the calculation of higher order corrections. The corresponding result in semi-inclusive DIS is also given for the comparison with Drell-Yan processes.

KeYwords: QCD Phenomenology

ArXiv EPRINT: 1409.2938 
In processes with a transversely polarized hadron in the initial state a Single Spin Asymmetry (SSA) can appear. This asymmetry is of particular interest in theory and experiment. Its existence requires nonzero absorptive part in scattering amplitudes and spin-flip interactions. For processes involving large scale, one can make theoretical predictions in terms of spin-dependent matrix elements in the framework of collinear factorization [1-5]. These matrix elements describe quark-gluon correlations in the transversely polarized hadron. Therefore, measuring SSA will reveal the correlations, hence the inner structure of hadrons.

The collinear factorization of SSA is of twist-3. In general factorizations beyond twist-2 can be nontrivial even at the leading order of $\alpha_{s}$. This is partly reflected by the fact that different results of SSA in the lepton angular distribution of Drell-Yan processes exist in literature. In this work, we make a study of twist-3 factorization of SSA at the leading order of $\alpha_{s}$. The purpose is not only to solve the discrepancy but also to explicitly show gauge invariance of QCD and QED in the relevant hadronic tensor. We will show that the QCD gauge invariance is realized in different ways for different contributions to SSA. The obtained hadronic tensor for SSA is explicitly gauge invariant of QED. The importance of showing gauge invariance of QCD and QED is not only for obtaining consistent results in theory, but also for simplifying the calculation of QCD corrections at certain level as we will show. For comparison with results for Drell-Yan processes we also give corresponding results for SSA in Semi-Inclusive DIS(SIDIS).

We consider the Drell-Yan process:

$$
h_{A}\left(P_{A}, s\right)+h_{B}\left(P_{B}\right) \rightarrow \gamma^{*}(q)+X \rightarrow \ell^{-}\left(k_{1}\right)+\ell^{+}\left(q-k_{1}\right)+X,
$$

where $h_{A}$ is a spin- $1 / 2$ hadron with the spin-vector $s$. To study the process it is convenient to use the light-cone coordinate system, in which a vector $a^{\mu}$ is expressed as $a^{\mu}=\left(a^{+}, a^{-}, \vec{a}_{\perp}\right)=\left(\left(a^{0}+a^{3}\right) / \sqrt{2},\left(a^{0}-a^{3}\right) / \sqrt{2}, a^{1}, a^{2}\right)$ and $a_{\perp}^{2}=\left(a^{1}\right)^{2}+\left(a^{2}\right)^{2}$. In this system we introduce two light-cone vectors $l^{\mu}=(1,0,0,0)$ and $n^{\mu}=(0,1,0,0)$. With the two vectors one can define the metric $g_{\perp}^{\mu \nu}$ and the totally anti-symmetric tensor $\epsilon_{\perp}^{\mu \nu}$ in the transverse space:

$$
g_{\perp}^{\mu \nu}=g^{\mu \nu}-n^{\mu} l^{\nu}-n^{\nu} l^{\mu}, \quad \epsilon_{\perp}^{\mu \nu}=\epsilon^{\alpha \beta \mu \nu} l_{\alpha} n_{\beta}, \quad \epsilon_{\perp}^{12}=-\epsilon_{\perp}^{21}=1 .
$$

We take a frame in which the momenta of hadrons and the spin $s$ are given by:

$$
P_{A}^{\mu} \approx\left(P_{A}^{+}, 0,0,0\right), \quad P_{B}^{\mu} \approx\left(0, P_{B}^{-}, 0,0\right), \quad s^{\mu}=s_{\perp}^{\mu}=\left(0,0, s_{\perp}^{1}, s_{\perp}^{2}\right) .
$$

We will look at the angular distribution in Collins-Soper frame [8]. The solid angle of the lepton is given by $\Omega=(\theta, \phi)$. For the angular distributions studied here, we take the $x$-axis as the direction of the transverse spin, i.e., $s_{\perp}^{\mu}=\left(0,0, s_{\perp}^{1}, 0\right)$. The 3 -momentum of the outgoing lepton $\ell^{-}$in Collins-Soper frame is given by $Q / 2(\sin \theta \cos \phi, \sin \theta \sin \phi, \cos \theta)$. The differential angular distribution can be written as:

$$
\frac{d \sigma}{d Q^{2} d \Omega}=\frac{\alpha^{2} e_{q}^{2}}{4 S Q^{4}} \int d^{4} q \delta\left(q^{2}-Q^{2}\right) W^{\mu \nu} L_{\mu \nu}
$$


where $L_{\mu \nu}$ is the leptonic tensor $2\left(2 k_{1}^{\mu}\left(q-k_{1}\right)^{\nu}+2 k_{1}^{\nu}\left(q-k_{1}\right)^{\mu}-g^{\mu \nu} Q^{2}\right) . \quad e_{q}$ is electric charge fraction of the quark. $S$ is the invariant mass $S=\left(P_{A}+P_{B}\right)^{2} . W^{\mu \nu}$ is the hadronic tensor defined as

$$
W^{\mu \nu}=\sum_{X} \int \frac{d^{4} x}{(2 \pi)^{4}} e^{i q \cdot x}\left\langle h_{A}\left(P_{A}, s_{\perp}\right), h_{B}\left(P_{B}\right)\left|\bar{\psi}(0) \gamma^{\nu} \psi(0)\right| X\right\rangle\left\langle X\left|\bar{\psi}(x) \gamma^{\mu} \psi(x)\right| h_{B}\left(P_{B}\right), h_{A}\left(P_{A}, s_{\perp}\right)\right\rangle .
$$

The invariant mass of the lepton pair is $Q^{2}$. The SSA in the angular distribution is defined as:

$$
A_{N}=\left(\frac{d \sigma\left(\vec{s}_{\perp}\right)}{d Q^{2} d \Omega}-\frac{d \sigma\left(-\vec{s}_{\perp}\right)}{d Q^{2} d \Omega}\right) /\left(\frac{d \sigma\left(\vec{s}_{\perp}\right)}{d Q^{2} d \Omega}+\frac{d \sigma\left(-\vec{s}_{\perp}\right)}{d Q^{2} d \Omega}\right) .
$$

In the collinear factorization, the spin-dependent part of the differential cross-section at the leading order of $\alpha_{s}$ can be factorized with the ETQS matrix elements [1-5]. They are defined with QCD operators of twist-3. Not all ETQS matrix elements are independent, among them there are relations $[6,7]$. One of the matrix elements is relevant in this work. It is defined as:

$$
T_{F}\left(x_{1}, x_{2}\right) \tilde{s}_{\perp}^{\mu}=g_{s} \int \frac{d \lambda_{1} d \lambda_{2}}{4 \pi} e^{-i \lambda_{2}\left(x_{2}-x_{1}\right) P_{A}^{+}-i \lambda_{1} x_{1} P_{A}^{+}}\left\langle P_{A}, \vec{s}_{\perp}\left|\bar{\psi}\left(\lambda_{1} n\right) \gamma^{+} G^{+\mu}\left(\lambda_{2} n\right) \psi(0)\right| P_{A}, \vec{s}_{\perp}\right\rangle
$$

with $\tilde{s}_{\perp}^{\mu}=\epsilon_{\perp}^{\mu \nu} s_{\perp \nu}$. In the definition we have suppressed the gauge links. In the light-cone gauge $n \cdot G=0$ they are units. In other gauges one needs to insert the product of gauge links like

$$
\mathcal{L}_{n}(x)=P \exp \left\{-i g_{s} \int_{-\infty}^{0} d \lambda n \cdot G(\lambda n+x)\right\}
$$

between operators to make the definition gauge invariant. In this work we will work in Feynman gauge.

The asymmetry $A_{N}$ has been studied in several works [9-18]. As mentioned, different results exist. For simplicity we will only consider the case where the process is initiated by an anti-quark from $h_{B}$. The different results can be given as

$$
\frac{1}{2} A_{N}^{[14,15,18]}=A_{N}^{[10-12]}=2 A_{N}^{[16,17]}=-\frac{\sin (2 \theta) \sin \phi}{Q\left(1+\cos ^{2} \theta\right)} \frac{\int d x d y T_{F}(x, x) \bar{q}(y) \delta\left(x y S-Q^{2}\right)}{\int d x d y q(x) \bar{q}(y) \delta\left(x y S-Q^{2}\right)}
$$

where $\bar{q}(y)$ is the anti-quark distribution of $h_{B}$ and the numbers in $[\cdots]$ 's are reference numbers. In [9] there is an additional contribution with the derivative of $T_{F}(x, x)$. It is noted that in the works except ours in [17] $A_{N}$ is derived with the diagram expansion at the hadron level. In [17] a different approach is taken by replacing $h_{A}$ with a multi-parton state and $h_{B}$ with an anti-quark. Then one can calculate $A_{N}$ and $T_{F}$ with these parton states separately to find the factorized form. Since the hadron $h_{A}$ is transversely polarized, one will get null results for $A_{N}$ and $T_{F}$ if one replaces $h_{A}$ with a single quark because of the helicity conservation of QCD. To obtain nonzero results, one has to use a multiparton state instead of the single quark $[19,20]$. This approach is useful to clarify some discrepancies in results about SSA, e.g., in the evolution of $T_{F}(x, x)$ [21]. 


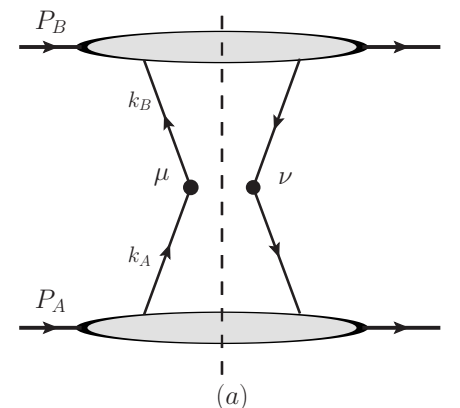

(a)

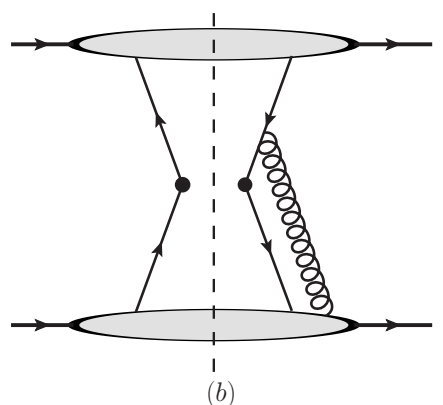

(b)

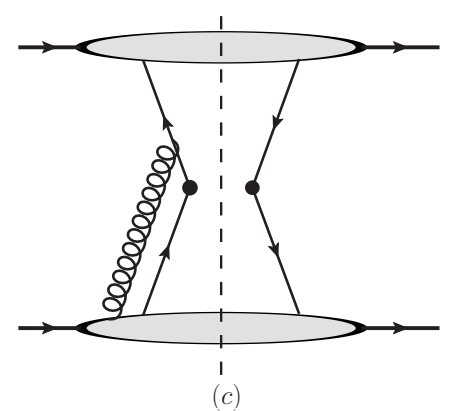

(c)

Figure 1. The tree-level diagrams for $W^{\mu \nu}$ of Drell-Yan processes. The black dots represent the insertion of the electromagnetic current.

In this work we will use the diagram expansion at hadron level to derive the hadronic tensor and $A_{N}$. At the leading order of $\alpha_{s}$ one has the contributions to $W^{\mu \nu}$ from diagrams given by figure 1 . These diagrams can be divided into three parts: the upper- and lower bubble, and the middle part given by explicit Feynman diagrams of parton scattering. The upper- and lower bubble represent jet-like Green functions related to the initial hadron $h_{B}$ and $h_{A}$, respectively. By imaging hadrons as bound states of quarks and gluons, these bubbles are the sum of all possible diagrams involving corresponding hadrons. Since they are jet-like, there are power counting for momenta of partons leaving or entering the bubbles. E.g., in figure $1 \mathrm{a}$ the momenta $k_{A}$ and $k_{B}$ scale like:

$$
k_{A}^{\mu} \sim Q\left(1, \lambda^{2}, \lambda, \lambda\right), \quad k_{B}^{\mu} \sim Q\left(\lambda^{2}, 1, \lambda, \lambda\right),
$$

with $\lambda \sim \Lambda_{Q C D} / Q$. For figure $1 \mathrm{~b}$ or $1 \mathrm{c}$, where a gluon leaves or enters bubbles, the gluon field vectors also scale like the pattern of the corresponding momentum as in eq. (10) in the gauge we work. In collinear factorization one needs to expand the contributions from figure 1 in power of $\lambda$. We will call the gluons with the polarization index - or + as $G^{-}$- and $G^{+}$-gluons. With the power counting one easily finds that the contributions from exchange of any number of $G^{-}$gluons with the upper bubble or $G^{+}$-gluons with the lower bubble can be at the same power of $\lambda$. The summation of the contributions is needed. In the first step, we only consider diagrams in figure 1.

In our case up to twist-3, we can always make the approximation by taking the twist2 part of the upper bubble of $h_{B}$ and neglecting all components of $k_{B}$ except the -component. The twist-2 part is given by the anti-quark distribution function $\bar{q}(y)$ of $h_{B}$ with $k_{B}^{-}=y P_{B}^{-}$. For momenta of partons from the lower bubble of $h_{A}$, one can always neglect the --components. With this in mind the contribution from figure 1a can be written as:

$$
\begin{aligned}
\left.W^{\mu \nu}\right|_{1 a}= & \int d^{3} \tilde{k}_{A} d k_{B}^{-}\left(\frac{1}{2 N_{c}} \bar{q}(y)\right)\left[\delta^{4}\left(k_{A}+k_{B}-q\right)\left(\gamma^{\nu} \gamma^{+} \gamma^{\mu}\right)_{j i}\right] \\
& \times\left[\int \frac{d^{3} \tilde{\xi}}{(2 \pi)^{3}} e^{-i \xi \cdot k_{A}}\left\langle h_{A}\left|\bar{\psi}_{j}(\xi) \psi_{i}(0)\right| h_{A}\right\rangle\right]
\end{aligned}
$$

with the notations:

$$
k_{B}^{\mu}=\left(0, k_{B}^{-}, 0,0\right), \quad k_{A}^{\mu}=\left(k_{A}^{+}, 0, \vec{k}_{A \perp}\right), \quad \xi^{\mu}=\left(0, \xi^{-}, \vec{\xi}_{\perp}\right), \quad d^{3} \tilde{\xi}=d \xi^{-} d^{2} \xi_{\perp}, \quad d^{3} \tilde{k}_{A}=d k_{A}^{+} d^{2} k_{A \perp} .
$$


$i j$ are the indices of Dirac spinor and color. In eq. (11) the term in $(\cdots)$ combined with $\gamma^{+}$ belongs to the twist-2 part of the upper bubble, the term in the first $[\cdots]$ without $\gamma^{+}$are from the middle part of figure 1a. The term in the second $[\cdots]$ is from the lower bubble. In the expansion of $\lambda$ one expands not only the middle part but also the bubbles in $\lambda$. The upper bubble is already expanded and the leading order is taken. The leading order of the lower bubble is given by taking the matrix as $\gamma^{-}$and keeping the +-component of $k_{A}$ as nonzero. One then obtains the standard result for the spin-independent part of $W^{\mu \nu}$ at twist-2:

$$
\left.W^{\mu \nu}\right|_{\text {twist }-2}=-\frac{1}{N_{c}} g_{\perp}^{\mu \nu} \delta^{2}\left(q_{\perp}\right) q(x) \bar{q}(y), \quad q^{+}=x P_{A}^{+}, \quad q^{-}=y P_{B}^{-} .
$$

It is noted that in this result the sum of contributions from exchange of any number of $G^{+}$ gluons with the lower bubble and $G^{-}$-gluons with the upper bubble has been performed. It results in that there are gauge links in the definition of the parton distribution functions $q(x)$ and $\bar{q}(y)[22]$.

It is clear that figure 1a also contains contributions at the next-to-leading order of $\lambda$ or twist-3, if we take the middle part or the lower bubble at the Next-to-Leading Order(NLO) of $\lambda$. To obtain the twist- 3 contribution it is convenient to write the product of $\Gamma$-matrices in eq. (11):

$$
\gamma^{\nu} \gamma^{+} \gamma^{\mu}=-\gamma^{+} g_{\perp}^{\mu \nu}+n^{\mu} \gamma_{\perp}^{\nu}+n^{\nu} \gamma_{\perp}^{\mu}+2 n^{\mu} n^{\nu} \gamma^{-}-i \epsilon^{\alpha \rho \mu \nu} \gamma_{5} \gamma_{\rho} n_{\alpha}
$$

In the above only the first three terms can give contributions at NLO of $\lambda$. For the contribution from the first term one needs to expand the middle part in $\lambda$. The NLO term in the expansion comes from the expansion of the $\delta$-function. We denote this contribution with the index $1 a^{+}$, which is given as:

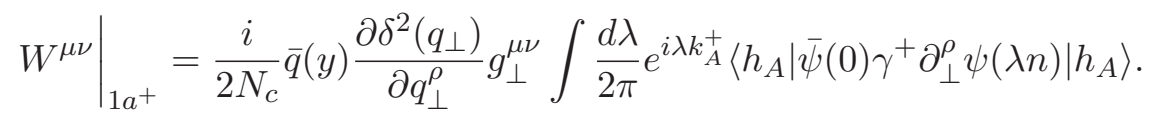

The contribution from the second- and third term in eq. (14) involves the matrix element of the operator $\bar{\psi}(\lambda n) \gamma_{\perp}^{\mu} \psi(0)$. As in the case of twist-2 contribution, the exchange of $G^{+}$gluons from the lower bubble will contribute at the same power of $\lambda$. These contributions need be summed with gauge links. After the summation, the contribution from $\gamma_{\perp}$ in eq. (14) involves the matrix element of $\bar{\psi}(\lambda n) \mathcal{L}_{n}(\lambda n) \gamma_{\perp}^{\mu} \mathcal{L}_{n}^{\dagger}(0) \psi(0)$. Since the $\gamma$-matrix here is $\gamma_{\perp}$, the contributions from exchanges of $G^{+}$-gluons is not included in the twist-3 contributions from figure $1 \mathrm{~b}$ and figure $1 \mathrm{c}$, where the involved $\gamma$-matrix is $\gamma^{+}$as shown later.

To find the twist-3 contributions from $\gamma_{\perp}$ in eq. (14) one needs to separate quark fields into the good- and bad component [23]:

$$
\psi=\psi_{+}+\psi_{-}, \quad \psi_{+}=\frac{1}{2} \gamma^{-} \gamma^{+} \psi, \quad \psi_{-}=\frac{1}{2} \gamma^{+} \gamma^{-} \psi,
$$

where $\psi_{+}$is the good component and $\psi_{-}$is the bad component. Using the equation of motion one can solve $\psi_{-}$in terms of $\psi_{+}$:

$$
\psi_{-}(\xi)=-\frac{1}{2} \mathcal{L}_{n}(\xi) \int_{-\infty}^{0} d \lambda \mathcal{L}_{n}^{\dagger}(\lambda n+\xi) \gamma^{+} \gamma_{\perp} \cdot D_{\perp} \psi_{+}(\lambda n+\xi) .
$$


With the solution the contribution from $\gamma_{\perp}$ is proportional to:

$$
\begin{aligned}
\int \frac{d \xi^{-}}{2 \pi} & e^{-i \xi^{-} k_{A}^{+}}\left\langle h_{A}\left|\bar{\psi}\left(\xi^{-} n\right) \mathcal{L}_{n}\left(\xi^{-} n\right) \gamma_{\perp}^{\mu} \mathcal{L}_{n}^{\dagger}(0) \psi(0)\right| h_{A}\right\rangle \\
= & -\frac{i}{2 k_{A}^{+}} \int \frac{d \xi^{-}}{2 \pi} e^{-i \xi^{-}} k_{A}^{+}\left[\left\langle h_{A}\left|\bar{\psi}\left(\xi^{-} n\right) \mathcal{L}_{n}\left(\xi^{-} n\right) \gamma_{\perp}^{\mu} \gamma^{+} \mathcal{L}_{n}^{\dagger}(0) \gamma_{\perp} \cdot D_{\perp} \psi(0)\right| h_{A}\right\rangle\right. \\
& \left.-\left\langle h_{A}\left|\overline{\left(\gamma_{\perp} \cdot D_{\perp} \psi\right)}\left(\xi^{-} n\right) \mathcal{L}_{n}\left(\xi^{-} n\right) \gamma^{+} \gamma_{\perp}^{\mu} \mathcal{L}_{n}^{\dagger}(0) \psi(0)\right| h_{A}\right\rangle\right]
\end{aligned}
$$

Using symmetries of Parity $(\mathrm{P})$ and Time-reversal( $\mathrm{T})$, one can show that the combination of matrix elements in $[\cdots]$ does not depend on the transverse spin $s_{\perp}$. The combination is zero. In the product $\mathcal{L}_{n}\left(\xi^{-} n\right) \mathcal{L}_{n}^{\dagger}(0)$ the direction of the gauge link $\mathcal{L}_{n}$ is pointing to the past as shown in eq. (8). It is noted that in the collinear factorization with non-singular gauge like Feynman gauge, it is equivalent by taking the direction of gauge links pointing to the future in the product. The reason for this is that one can use the products of the gauge links to form a gauge link along a closed contour and the enclosed area is zero. In fact, in non-singular gauges one can show with PT-symmetry the contribution in eq. (15) is also zero. Therefore, we conclude that the total contribution from figure 1a is zero in non-singular gauges.

The contributions from figure $1 \mathrm{~b}$ and figure $1 \mathrm{c}$ after the approximation made similarly for eq. (11) are:

$$
\begin{aligned}
& \left.W^{\mu \nu}\right|_{1 c}=\int d^{3} \tilde{k}_{A} d^{3} \tilde{k} d k_{B}^{-} \frac{1}{2 N_{c}} \bar{q}(y)\left[\delta^{4}\left(k_{A}+k+k_{B}-q\right) \gamma^{\nu} \gamma^{+} \gamma_{\rho} \frac{\gamma \cdot\left(-k-k_{B}\right)}{\left(k+k_{B}\right)^{2}+i \varepsilon} \gamma^{\mu}\right]_{j i} \mathcal{M}_{i j}^{\rho}\left(k_{A}, k\right), \\
& \left.W^{\mu \nu}\right|_{1 b}=\int d^{3} \tilde{k}_{A} d^{3} \tilde{k} d k_{B}^{-} \frac{1}{2 N_{c}} \bar{q}(y)\left[\delta^{4}\left(k_{A}+k_{B}-q\right) \gamma^{\nu} \frac{\gamma \cdot\left(k-k_{B}\right)}{\left(k-k_{B}\right)^{2}-i \varepsilon} \gamma_{\rho} \gamma^{+} \gamma^{\mu}\right]_{j i} \mathcal{M}_{i j}^{\rho}\left(k_{A}, k\right),
\end{aligned}
$$

with the quark-gluon correlator

$$
\mathcal{M}_{i j}^{\rho}\left(k_{A}, k\right)=\left.g_{s} \int \frac{d \xi_{1}^{-} d^{2} \xi_{1 \perp} d \xi_{2}^{-} d^{2} \xi_{2 \perp}}{(2 \pi)^{6}} e^{i \xi_{1} \cdot k_{A}+i \xi_{2} \cdot k}\left\langle h_{A}\left|\bar{\psi}_{j}(0)\left[G^{\rho}\left(\xi_{2}\right) \psi\left(\xi_{1}\right)\right]_{i}\right| h_{A}\right\rangle\right|_{\xi_{1,2}^{+}=0},
$$

where $k$ is the momentum of the gluon. It is given by $k^{\mu}=\left(k^{+}, 0, \vec{k}_{\perp}\right)$ and the measure $d^{3} \tilde{k}=d k^{+} d^{2} k_{\perp} . i j$ are the indices of Dirac spinor and color. In eq. (19) the terms in $[\cdots]$ are from the middle part of diagrams, and $\mathcal{M}^{\rho}$ is for the lower bubble. To find the relevant contributions one can represent the matrix $\mathcal{M}^{\rho}$ as:

$$
\mathcal{M}^{\rho}\left(k_{A}, k\right)=\frac{1}{2 N_{c}}\left(M^{\rho}\left(k_{A}, k\right) \gamma^{-}+M_{A}^{\rho}\left(k_{A}, k\right) \gamma_{5} \gamma^{-}\right)+\cdots,
$$

the $\cdots$ represent terms which will give contributions beyond twist-3. The term with the matrix of $\gamma_{5} \gamma^{-}$gives no contribution at leading order of $\alpha_{s}$. Here the involved operator is with $\gamma^{+}$as mentioned after eq. (15). If we expand the contributions in eq. (19) in $\lambda$, the contributions at the leading order or at twist-2 are spin-independent and only contain $M^{+}$. These contributions are summed in eq. (13) as gauge links in $q(x)$.

At NLO of $\lambda$ or at twist-3 the contributions can be spin-dependent. To obtain the contributions at twist-3 one needs to expand the middle parts in eq. (19) at NLO and the lower bubble $\mathcal{M}^{\rho}$ at NLO. One should note that in the expansion of the middle part the 
$\delta$-function depends on $k_{A \perp}$ and $k_{\perp}$. This dependence also needs to be expanded. With the power counting for the gluon field explained after eq. (10) $M^{\rho}$ has the NLO contribution with $\rho=\perp$. Keeping these in mind the expansion is straightforward. We obtain the contributions from figure $1 \mathrm{~b}$ and figure $1 \mathrm{c}$ as:

$$
\begin{aligned}
& \left.W^{\mu \nu}\right|_{1 c}=\frac{1}{N_{c}} \bar{q}(y) \int d k^{+}\left(\frac{i}{k^{+}+i \varepsilon}\right)\left\{i \frac{\partial \delta^{2}\left(q_{\perp}\right)}{\partial q_{\perp}^{\rho}} g_{\perp}^{\mu \nu} \int d^{2} k_{A \perp} d^{2} k_{\perp}\left(k_{A \perp}^{\rho}+k_{\perp}^{\rho}\right) M^{+}\left(k_{A}-k^{+} l, k\right)\right. \\
& \left.+\delta^{2}\left(q_{\perp}\right) \frac{l^{\mu}}{k_{B}^{-}}\left[g_{s} \int \frac{d \xi_{1}^{-} d \xi_{2}^{-}}{2(2 \pi)^{2}} e^{i \xi_{1}^{-}\left(k_{A}^{+}-k^{+}\right)+i \xi_{2}^{-} k^{+}}\left\langle h_{A}\left|\bar{\psi}(0) \gamma^{+} \hat{G}^{+\nu}\left(\xi_{2}^{-} n\right) \psi\left(\xi_{1}^{-} n\right)\right| h_{A}\right\rangle\right]\right\}+\cdots, \\
& \left.W^{\mu \nu}\right|_{1 b}=\frac{1}{N_{c}} \bar{q}(y) \int d k^{+}\left(\frac{-i}{-k^{+}-i \varepsilon}\right)\left\{-i \frac{\partial \delta^{2}\left(q_{\perp}\right)}{\partial q_{\perp}^{\rho}} g_{\perp}^{\mu \nu} \int d^{2} k_{A \perp} d^{2} k_{\perp} k_{A \perp}^{\rho} M^{+}\left(k_{A}, k\right)\right. \\
& \left.+\delta^{2}\left(q_{\perp}\right) \frac{l^{\nu}}{k_{B}^{-}}\left[g_{s} \int \frac{d \xi_{1}^{-} d \xi_{2}^{-}}{2(2 \pi)^{2}} e^{i \xi_{1}^{-} k_{A}^{+}+i \xi_{2}^{-} k^{+}}\left\langle h_{A}\left|\bar{\psi}(0) \gamma^{+} \hat{G}^{+\mu}\left(\xi_{2}^{-} n\right) \psi\left(\xi_{1}^{-} n\right)\right| h_{A}\right\rangle\right]\right\}+\cdots,
\end{aligned}
$$

with

$$
\hat{G}^{\mu \nu}=\partial^{\mu} G^{\nu}-\partial^{\nu} G^{\mu}, \quad k_{A}^{+}=q^{+}=x P_{A}^{+}, \quad k_{B}^{-}=q^{-}=y P_{B}^{-} .
$$

In eq. (22) $\cdots$ stand for higher order of $\lambda$ or spin-independent parts.

The results in eq. (22) are not expressed with matrix elements of gauge invariant operators. E.g., in the second- and fourth line one has the operator $\hat{G}^{\mu \nu}$ which is not exactly the gluon field strength tensor $G^{\mu \nu}$. It is commonly believed that one will have the results expressed with gauge invariant operators after summing of any number of gluon exchanges additionally to the exchange of one gluon in figure $1 \mathrm{~b}$ and figure $1 \mathrm{c}$. This gives a common rule for obtaining gauge invariant results: one only calculates figure $1 \mathrm{~b}$ and figure 1c with the exchange of a $G^{+}$-gluon in Feynman gauge and obtains the combination at NLO of $\lambda k_{\perp}^{\mu} G^{+}$. Then one simply makes the replacement $k_{\perp}^{\mu} G^{+} \rightarrow G^{+\mu}$ in the results, e.g., in $[12,16]$. Although the replacement will produce correct results here, but this rule is not fully augmented to be correct. This brings up the question if the results are really QCD gauge invariant.

Taking the contributions of figure $1 \mathrm{c}$ as an example, one indeed obtains for the contribution with $\delta^{2}\left(q_{\perp}\right)$ the full gluon field strength tensor $G^{+\mu}$ instead of $\hat{G}^{+\mu}$ and gauge links between operators, after the summation of any number of gluon exchanges. After taking the cut of the quark propagator in the middle part of figure 1c, one obtains the contribution expressed with $T_{F}(x, x)$. But for the contribution with the derivative of $\delta^{2}\left(q_{\perp}\right)$ the exchanged gluons are all $G^{+}$-gluons. The contribution from the exchange of one or more gluons with the transverse polarization is beyond the order of $\lambda$ considered here. Therefore, one can not use the common rule in a direct way to obtain a gauge invariant result from this contribution. In fact the contribution from the exchange of $G^{+}$-gluons can be easily summed with gauge links. The summation is like that in the factorization at twist-2. Therefore, after the summation of $n$-gluon exchanges with $n>0$, we have the sum of figure $1 \mathrm{~b}$ and figure $1 \mathrm{c}$ :

$$
\begin{aligned}
\left.W^{\mu \nu}\right|_{1 b+1 c}= & \frac{1}{2 N_{c} k_{B}^{-}} \delta^{2}\left(q_{\perp}\right)\left(l^{\mu} \tilde{s}_{\perp}^{\nu}+l^{\nu} \tilde{s}_{\perp}^{\mu}\right) \bar{q}(y) T_{F}(x, x)+\frac{i}{N_{c}} g_{\perp}^{\mu \nu} \bar{q}(y) \frac{\partial \delta^{2}\left(q_{\perp}\right)}{\partial q_{\perp}^{\rho}} \int \frac{d \lambda}{4 \pi} e^{i k_{A}^{+} \lambda} \\
& {\left[\left\langle h_{A}\left|\bar{\psi}(0) \mathcal{L}_{n}(0) \gamma^{+} \partial_{\perp}^{\rho}\left(\mathcal{L}_{n}^{\dagger}(\lambda n) \psi(\lambda n)\right)\right| h_{A}\right\rangle-\left\langle h_{A}\left|\bar{\psi}(0) \gamma^{+} \partial_{\perp}^{\rho} \psi(\lambda n)\right| h_{A}\right\rangle\right] . }
\end{aligned}
$$


In a non-singular gauge which we use the first term in $[\cdots]$ is gauge invariant. The second term in $[\cdots]$ is not gauge invariant, but it will be canceled in the sum with the contribution in eq. (15). In non-singular gauges it is zero.

To simplify our result in the above, we note that one can derive the identity in nonsingular gauges:

$$
\partial_{\perp}^{\mu}\left(\mathcal{L}_{n}^{\dagger} \psi\right)(\xi)=\mathcal{L}_{n}^{\dagger} D_{\perp}^{\mu} \psi(\xi)-i g_{s} \int_{-\infty}^{0} d \lambda\left(\mathcal{L}_{n}^{\dagger} G^{+\mu} \mathcal{L}_{n}\right)(\lambda n+\xi)\left(\mathcal{L}_{n}^{\dagger} \psi\right)(\xi)
$$

Using this identity and PT-symmetry we can derive our final result of the twist-3 contribution of $W^{\mu \nu}$ from figure 1 as:

$$
\left.W^{\mu \nu}\right|_{\text {Fig. } 1}=\frac{1}{2 N_{c}} \bar{q}(y) T_{F}(x, x)\left[\frac{1}{q^{-}} \delta^{2}\left(q_{\perp}\right)\left(l^{\mu} \tilde{s}_{\perp}^{\nu}+l^{\nu} \tilde{s}_{\perp}^{\mu}\right)+g_{\perp}^{\mu \nu} \frac{\partial \delta^{2}\left(q_{\perp}\right)}{\partial q_{\perp}^{\rho}} \tilde{s}_{\perp}^{\rho}\right] .
$$

This result is QCD gauge invariant. From our derivation one can see that the way to obtain final results with matrix elements of QCD gauge invariant operators is different for different contributions, although with the common rule mentioned before one obtains the same results. In the summation of exchanges of gluons for the contribution with $\delta^{2}\left(q_{\perp}\right)$, one of the exchanged gluons is transversely polarized. This enables us immediately to obtain the matrix element of the gauge invariant operator with $G^{+\mu}$. In the summation of exchanges of gluons for the contribution with the derivative of $\delta^{2}\left(q_{\perp}\right)$, all exchanged gluons are $G^{+}-$ gluons, the summed form does not involve $G^{+\mu}$ as shown in eq. (24). Only after using the identity and PT-symmetry, one can express the contribution with $T_{F}(x, x)$. This has a consequence which we will discuss after that we have given the relevant results for SIDIS.

In the contribution of figure $1, T_{F}(x, x)$ is defined with the chirality-even operator. There is also a twist-3 contribution involving matrix elements defined with chirality-odd operators [16]. One of the involved distributions defined with chirality-odd operators is the transversity distribution of $h_{A}$ :

$$
h_{1}(x) s_{\perp}^{\mu}=\int \frac{d \xi^{-}}{4 \pi} e^{-i x \xi^{-} P_{A}^{+}}\left\langle h_{A}\left|\bar{q}\left(\xi^{-} n\right) \gamma_{5} \gamma^{+} \gamma_{\perp}^{\mu} q(0)\right| h_{A}\right\rangle .
$$

Another one is the chirality-odd quark-gluon correlation of $h_{B}$ defined as:

$$
T_{F}^{(\sigma)}\left(y_{1}, y_{2}\right)=-g_{s} \int \frac{d \xi_{1}^{+} d \xi_{2}^{+}}{4 \pi} e^{-i \xi_{1}^{+} y_{1} P_{B}^{-}-i \xi_{2}^{+}\left(y_{2}-y_{1}\right) P_{B}^{-}}\left\langle h_{B}\left|\bar{q}(0)\left(i \gamma_{\perp \mu} \gamma^{-}\right) G^{+\mu}\left(\xi_{2}^{+} l\right) q\left(\xi_{1}^{+} l\right)\right| h_{B}\right\rangle
$$

In the above we have suppressed gauge links. The chirality-odd contribution to $W^{\mu \nu}$ can be derived in a similar way. Adding this contribution we have the complete result for the spin-dependent part of $W^{\mu \nu}$ :

$$
\begin{gathered}
W^{\mu \nu}=\frac{1}{2 N_{c}}\left\{-T_{F}^{(\sigma)}(y, y) h_{1}(x)\left[\frac{1}{2} \frac{\partial \delta^{2}\left(q_{\perp}\right)}{\partial q_{\perp}^{\rho}}\left(g_{\perp}^{\mu \rho} \tilde{s}_{\perp}^{\nu}+g_{\perp}^{\nu \rho} \tilde{s}_{\perp}^{\mu}-g_{\perp}^{\mu \nu} \tilde{s}_{\perp}^{\rho}\right)+\frac{\delta^{2}\left(q_{\perp}\right)}{P_{B} \cdot q}\left(P_{B}^{\mu} \tilde{s}_{\perp}^{\nu}+P_{B}^{\nu} \tilde{s}_{\perp}^{\mu}\right)\right]\right. \\
\left.+\bar{q}(y) T_{F}(x, x)\left[\frac{\delta^{2}\left(q_{\perp}\right)}{P_{A} \cdot q}\left(P_{A}^{\mu} \tilde{s}_{\perp}^{\nu}+P_{A}^{\nu} \tilde{s}_{\perp}^{\mu}\right)+g_{\perp}^{\mu \nu} \frac{\partial \delta^{2}\left(q_{\perp}\right)}{\partial q_{\perp}^{\rho}} \tilde{s}_{\perp}^{\rho}\right]\right\}+\mathcal{O}\left(\alpha_{s}\right) .
\end{gathered}
$$


With the explicit result for $W^{\mu \nu}$ one can check if it is $\mathrm{U}(1)$-gauge invariant. However, one can not simply contract $q_{\mu}$ with $W^{\mu \nu}$ here, because $W^{\mu \nu}$ contains $\delta^{2}\left(q_{\perp}\right)$ and its derivative. One should take $W^{\mu \nu}$ as a distribution tensor. The $\mathrm{U}(1)$-gauge invariance should be understood in the sense of integration of $q_{\perp}$. Therefore, the U(1)-gauge invariance implies:

$$
\int d^{2} q_{\perp} \mathcal{F}\left(q_{\perp}\right) W^{\mu \nu} q_{\mu}=0
$$

with $\mathcal{F}\left(q_{\perp}\right)$ as a test function. Our result satisfies this equation.

From our result of $W^{\mu \nu}$ we can calculate the asymmetry $A_{N}$. The result is with $s_{\perp}^{\mu}=(0,0,1,0):$

$$
A_{N}=-\frac{\sin (2 \theta) \sin \phi}{2 Q\left(1+\cos ^{2} \theta\right)} \frac{\int d x d y\left[T_{F}(x, x) \bar{q}(y)+h_{1}(x) T_{F}^{(\sigma)}(y, y)\right] \delta\left(x y S-Q^{2}\right)}{\int d x d y q(x) \bar{q}(y) \delta\left(x y S-Q^{2}\right)} .
$$

This result agrees with that given in [16]. Especially, the chiral-even contribution agrees with our early result in [17]. Since the same contribution has been derived in a completely different way, we believe that the discrepancy illustrated in eq. (9) is solved with eq. (31).

From our result $W^{\mu \nu}$ consists of different tensor structures at leading order of $\alpha_{s}$. This enables us to construct more observables. In the differential cross-section defined in eq. (4) $q_{\perp}$ is integrated. In the integration one can introduce some weight functions to have the so-called weighted SSA. In the case here, we can introduce two weighted SSA's. We derive:

$$
\begin{aligned}
\frac{d \sigma\left\langle q_{\perp} \cdot \tilde{s}_{\perp}\right\rangle}{d Q^{2} d \Omega} & =\frac{\alpha^{2} e_{q}^{2}}{4 S Q^{4}} \int d^{4} q \delta\left(q^{2}-Q^{2}\right)\left(q_{\perp} \cdot \tilde{s}_{\perp}\right) W^{\mu \nu} L_{\mu \nu} \\
& =-\frac{\alpha^{2} e_{q}^{2}}{8 N_{c} Q^{2}} \int d x d y \delta\left(x y S-Q^{2}\right)\left[\left(1+\cos ^{2} \theta\right) \bar{q}(y) T_{F}(x, x)-\frac{1}{2} \sin ^{2} \theta \cos (2 \phi) h_{1}(x) T_{F}^{(\sigma)}(y, y)\right] \\
\frac{d \sigma\left\langle q_{\perp} \cdot s_{\perp}\right\rangle}{d Q^{2} d \Omega} & =\frac{\alpha^{2} e_{q}^{2}}{4 S Q^{4}} \int d^{4} q \delta\left(q^{2}-Q^{2}\right)\left(q_{\perp} \cdot s_{\perp}\right) W^{\mu \nu} L_{\mu \nu} \\
& =-\frac{\alpha^{2} e_{q}^{2}}{16 N_{c} Q^{2}} \sin ^{2} \theta \sin (2 \phi) \int d x d y \delta\left(x y S-Q^{2}\right) h_{1}(x) T_{F}^{(\sigma)}(y, y) .
\end{aligned}
$$

Measuring these observables and $A_{N}$ will help to disentangle the chiral-even- and chiral-odd contribution.

One can expect that there is a close relation between Drell-Yan process and SIDIS. To compare SSA in the two processes, we discuss here SSA in SIDIS briefly. We study here SSA in Semi-Inclusive DIS(SIDIS):

$$
\ell(k)+h_{A}(P, s) \rightarrow \ell\left(k^{\prime}\right)+h_{B}\left(P_{h}\right)+X
$$

where the initial hadron is polarized with the spin vector $s$. We take the initial electron as unpolarized. The polarization of particles in the final state is not observed or summed. The standard variables for SIDIS are defined as:

$$
x_{B}=\frac{Q^{2}}{2 P \cdot q}, \quad y=\frac{P \cdot q}{P \cdot k}, \quad z_{h}=\frac{P \cdot P_{h}}{P \cdot q} .
$$




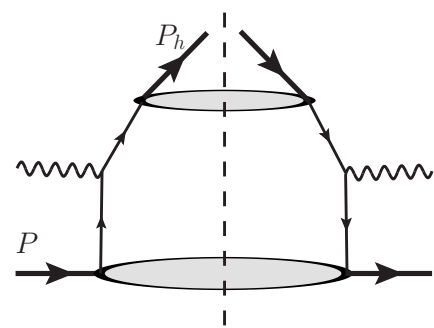

(a)

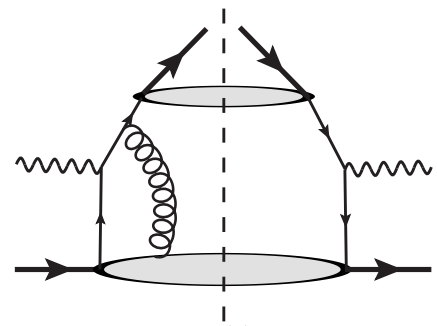

(b)

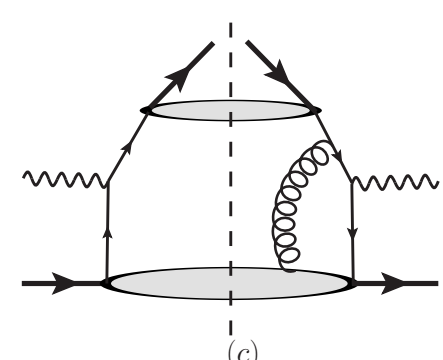

(c)

Figure 2. Diagrams for contributions in SIDIS.

The hadronic tensor of SIDIS is defined as

$$
W^{\mu \nu}=\sum_{X} \int \frac{d^{4} x}{(2 \pi)^{4}} e^{i q \cdot x}\left\langle h_{A}\left|J^{\mu}(x)\right| h_{B}, X\right\rangle\left\langle X, h_{B}\left|J^{\nu}(0)\right| h_{A}\right\rangle .
$$

At leading order of $\alpha_{s}$, the spin-dependent part of $W^{\mu \nu}$ receives contributions from diagrams given in figure 2. To analyze SIDIS, it is convenient to take the frame in which the initial hadron moves in the $z$-direction and the final hadron moves in the $-z$-direction. The transverse space is then defined with $P$ and $P_{h}$. In this frame the virtual photon has a transverse momentum. The result obtained in this frame can be expressed in a covariant way. The calculation of figure 2 is similar to that of figure 1 for Drell-Yan processes. We will skip any detail of the calculation and give the results in the following.

From figure 2 one can derive the spin-dependent part of $W^{\mu \nu}$ as:

$$
W^{\mu \nu}=\frac{1}{z_{h}} d\left(z_{h}\right) T_{F}\left(x_{B}, x_{B}\right)\left[\delta^{2}\left(q_{\perp}\right) \frac{1}{P \cdot q}\left(P^{\mu} \tilde{s}_{\perp}^{\nu}+P^{\nu} \tilde{s}_{\perp}^{\mu}\right)+g_{\perp h}^{\mu \nu} \tilde{s}_{\perp h}^{\rho} \frac{\partial \delta^{2}\left(q_{\perp}\right)}{\partial q_{\perp}^{\rho}}\right]
$$

with

$$
g_{\perp h}^{\mu \nu}=g^{\mu \nu}-\frac{1}{P \cdot P_{h}}\left(P^{\mu} P_{h}^{\nu}+P^{\nu} P_{h}^{\mu}\right), \quad \tilde{s}_{\perp h}^{\mu}=\frac{1}{P \cdot P_{h}} \epsilon^{\alpha \beta \mu \nu} P_{\alpha} P_{h \beta} s_{\nu}, \quad q_{\perp}^{\mu}=g_{\perp h}^{\mu \nu} q_{\nu} .
$$

$d\left(z_{h}\right)$ is the quark fragmentation function of $h_{B}$. It is defined:

$$
d(z)=z \int \frac{d y}{4 \pi} e^{-i y P_{h}^{-} / z} \sum_{X} \frac{1}{2 N_{c}} \operatorname{Tr}\left[\left\langle 0\left|\gamma^{+} \psi(0)\right| h\left(P_{h}\right) X\right\rangle\left\langle X h\left(P_{h}\right)|\bar{\psi}(y l)| 0\right\rangle\right],
$$

where we have suppressed gauge links. In the definition, the hadron moves in the $-z$ direction. In SIDIS $W^{\mu \nu}$ does not receive contributions involving matrix elements defined with chirality-odd operators. This is because the chirality-odd quark-gluon fragmentation function $\hat{T}_{F}^{(\sigma)}\left(z_{1}, z_{2}\right)$, which corresponds to the chirality-odd quark-gluon distribution $T_{F}^{(\sigma)}\left(x_{1}, x_{2}\right)$ defined in eq. (28), is zero at $z_{1}=z_{2}[24]$. Beyond the tree level $W^{\mu \nu}$ can have chirality-odd contributions.

At first look, the spin-dependent part of $W^{\mu \nu}$ in eq. (36) has also two terms corresponding to the chirality-even contribution in eq. (26), (29) for Drell-Yan process. But, one can express eq. (36) with the metric $g^{\mu \nu}$ of the transverse space defined with $P$ and $q$. 
The quantities in the two transverse spaces are given by:

$$
\begin{array}{ll}
g_{\perp}^{\mu \nu}=g^{\mu \nu}-\frac{1}{P \cdot q}\left(P^{\mu}\left(x_{B} P+q\right)^{\nu}+P^{\nu}\left(x_{B} P+q\right)^{\mu}\right), & P_{h \perp}^{\mu \nu}=g_{\perp}^{\mu \nu} P_{h \nu}=-z_{h} q_{\perp}^{\mu}+\mathcal{O}\left(P_{h \perp}^{2}\right), \\
g_{\perp}^{\mu \nu}=g_{\perp h}^{\mu \nu}+\frac{1}{z_{h} P \cdot q}\left(P^{\mu} P_{h \perp}^{\nu}+P^{\nu} P_{h \perp}^{\mu}\right)+\mathcal{O}\left(P_{h \perp}^{2}\right), & \tilde{s}_{\perp}^{\mu}=\frac{1}{P \cdot q} \epsilon^{\alpha \beta \mu \nu} P_{\alpha} q_{\beta} s_{\nu}=\tilde{s}_{\perp h}^{\mu} .
\end{array}
$$

Here we assume that the spin vector $s$ in eq. (33) is in the transverse space defined with $P$ and $q$. Using these relations one can re-write $W^{\mu \nu}$ in eq. (36) as:

$$
W^{\mu \nu}=-z_{h}^{2} d\left(z_{h}\right) T_{F}\left(x_{B}, x_{B}\right) g_{\perp}^{\mu \nu} \tilde{s}_{\perp}^{\rho} \frac{\partial \delta^{2}\left(P_{\perp h}\right)}{\partial P_{h \perp}^{\rho}}+\mathcal{O}\left(\alpha_{s}\right) .
$$

This result is explicitly $\mathrm{U}(1)$-gauge invariant. In comparison with $W^{\mu \nu}$ of Drell-Yan processes, $W^{\mu \nu}$ of SIDIS has only one term which is spin-dependent. Therefore, one can only construct one weighted SSA weighted with $P_{h \perp} \cdot \tilde{s}_{\perp}$ in the integration over $P_{h \perp}$. The result is:

$$
\frac{d \sigma\left\langle P_{h \perp} \cdot \tilde{s}_{\perp}\right\rangle}{d x_{B} d y d z_{h}}=\frac{\pi \alpha^{2} e_{q}^{2}}{Q^{2}} \frac{1+(1-y)^{2}}{y} z_{h} d\left(z_{h}\right) T_{F}\left(x_{B}, x_{B}\right)+\mathcal{O}\left(\alpha_{s}\right) .
$$

This result agrees with that given in [25]. In [25] one uses Transverse-MomentumDependent (TMD) factorization and the weighted SSA is expressed with the transverse momentum moment of Sivers function. The moment is related to $T_{F}(x, x)$ at tree-level.

We have shown that how QCD gauge invariance for each term of $W^{\mu \nu}$ in eq. (26) for Drell-Yan processes is made. For the term with the derivative of $\delta^{2}\left(q_{\perp}\right)$ we have to sum all exchanges of $G^{+}$-gluons into gauge links as shown in eq. (24). This fact gives us the opportunity to simplify the calculation of corrections from higher order of $\alpha_{s}$. It is noted that the derivative of the $\delta$-function for the momentum conservation comes from the expansion of transverse momenta of partons. In calculating the twist- 3 contribution with the derivative of the $\delta$-function, one can already neglect the transverse momenta of partons except those in the $\delta$-function. In this case, one essentially deals with the amplitude of the annihilation of an on-shell quark-antiquark pair combined with $G^{+}$-gluons from the lower bubble in figure 1 into the virtual photon. Now the summation of all exchanges of $G^{+}$-gluons can be easily performed. After the summation the contribution with the derivative only comes from figure 1a but with the bubbles defined with insertion of gauge links. Because of this, the virtual correction for the term with the derivative of $\delta^{2}\left(q_{\perp}\right)$ is completely determined by the quark form factor with the subtraction defined with the gauge links. The situation is similar to that of corrections beyond tree-level in TMD factorization discussed in [26]. We note here that in the subtraction with the gauge links the collinear divergences will be subtracted. The divergences from soft gluons are expected to be cancelled in twist-3 factorization [27].

It is noted that some weighted SSA observables do not receive contributions from the part of $W^{\mu \nu}$ with $\delta^{2}\left(q_{\perp}\right)$. They receive contributions from the part with the derivative of $\delta^{2}\left(q_{\perp}\right)$ and the part with $q_{\perp} \neq 0$. From the above discussion, the virtual correction of these weighted SSA observables is determined by the quark form factor. This will simplify 
the calculation of the virtual correction, because the virtual correction from exchanges of gluons in the left part of figure $1 \mathrm{~b}$ is more complicated than that from exchanges of gluons in the right part of figure 1b. But, for the part with the derivative of $\delta^{2}\left(q_{\perp}\right)$ the two corrections are same. The same conclusion can also be made for SIDIS. The one-loop calculation of the virtual correction involving $T_{F}$ for Drell-Yan processe in [28] and for SIDIS in [29] verifies our conclusion explicitly.

To summarize: we have studied collinear factorization at twist-3 of the hadronic tensor $W^{\mu \nu}$ relevant for SSA in Drell-Yan processes with the emphasis of how to obtain results respecting gauge invariance of $\mathrm{QCD}$, and with the hope to solve the discrepancy of SSA in the lepton angular distribution $A_{N}$. Our result for $A_{N}$ agrees our early result in [17]. It is noted that the result in [17] is derived in an approach different that that in this work. A by-product with our result of $W^{\mu \nu}$ is that we can construct another two observables of SSA in addition to $A_{N}$. Measuring these observables will help to disentangle different contributions in $W^{\mu \nu}$. We also give the result of $W^{\mu \nu}$ relevant for SSA in SIDIS, where one can only obtain one SSA observable. We have shown that the QCD gauge invariance for different contributions in $W^{\mu \nu}$ is made in different ways. In showing this, we obtain an interesting result that the virtual correction to one structure function of $W^{\mu \nu}$, hence to some weighted SSA observables, is completely determined by the quark form factor with subtractions. This will simplify higher order corrections.

\section{Acknowledgments}

The work of J.P. Ma is supported by National Nature Science Foundation of P.R. China (No. 11275244). The support from CAS center for excellence in particle physics is acknowledged.

Open Access. This article is distributed under the terms of the Creative Commons Attribution License (CC-BY 4.0), which permits any use, distribution and reproduction in any medium, provided the original author(s) and source are credited.

\section{References}

[1] A.V. Efremov and O.V. Teryaev, On Spin Effects in Quantum Chromodynamics (in Russian), Sov. J. Nucl. Phys. 36 (1982) 140 [inSPIRE].

[2] A.V. Efremov and O.V. Teryaev, QCD Asymmetry and Polarized Hadron Structure Functions, Phys. Lett. B 150 (1985) 383 [InSPIRE].

[3] J.-w. Qiu and G.F. Sterman, Single transverse spin asymmetries, Phys. Rev. Lett. 67 (1991) 2264 [INSPIRE].

[4] J.-w. Qiu and G.F. Sterman, Single transverse spin asymmetries in direct photon production, Nucl. Phys. B 378 (1992) 52 [InSPIRE].

[5] J.-w. Qiu and G.F. Sterman, Single transverse spin asymmetries in hadronic pion production, Phys. Rev. D 59 (1999) 014004 [hep-ph/9806356] [INSPIRE]. 
[6] H. Eguchi, Y. Koike and K. Tanaka, Single Transverse Spin Asymmetry for Large- $p_{T}$ Pion Production in Semi-Inclusive Deep Inelastic Scattering, Nucl. Phys. B 752 (2006) 1 [hep-ph/0604003] [INSPIRE].

[7] J. Zhou, F. Yuan and Z.-T. Liang, Transverse momentum dependent quark distributions and polarized Drell-Yan processes, Phys. Rev. D 81 (2010) 054008 [arXiv:0909.2238] [INSPIRE].

[8] J.C. Collins and D.E. Soper, Angular Distribution of Dileptons in High-Energy Hadron Collisions, Phys. Rev. D 16 (1977) 2219 [InSPIRE].

[9] N. Hammon, O. Teryaev and A. Schafer, Single spin asymmetry for the Drell-Yan process, Phys. Lett. B 390 (1997) 409 [hep-ph/9611359] [INSPIRE].

[10] D. Boer, P.J. Mulders and O.V. Teryaev, Single spin asymmetries from a gluonic background in the Drell-Yan process, Phys. Rev. D 57 (1998) 3057 [hep-ph/9710223] [INSPIRE].

[11] D. Boer and P.J. Mulders, Color gauge invariance in the Drell-Yan process, Nucl. Phys. B 569 (2000) 505 [hep-ph/9906223] [INSPIRE].

[12] D. Boer and J.-w. Qiu, Single transverse spin asymmetry in Drell-Yan lepton angular distribution, Phys. Rev. D 65 (2002) 034008 [hep-ph/0108179] [InSPIRE].

[13] J.P. Ma and Q. Wang, On unique predictions for single spin azimuthal asymmetry, Eur. Phys. J. C 37 (2004) 293 [hep-ph/0310245] [INSPIRE].

[14] I.V. Anikin and O.V. Teryaev, Gauge invariance, causality and gluonic poles, Phys. Lett. B 690 (2010) 519 [arXiv:1003.1482] [INSPIRE].

[15] I.V. Anikin and O.V. Teryaev, Asymmetries associated with higher twists: gauge invariance, gluonic poles and twist three, arXiv:1201.2569 [INSPIRE].

[16] J. Zhou and A. Metz, Transverse single spin asymmetry in the Drell-Yan process, Phys. Rev. D 86 (2012) 014001 [arXiv:1011.5871] [InSPIRE].

[17] J.P. Ma and G.P. Zhang, Single Spin Asymmetry in Lepton Angular Distribution of Drell-Yan Processes, JHEP 11 (2012) 156 [arXiv:1203.6415] [INSPIRE].

[18] G. Re Calegari and P.G. Ratcliffe, Single Transverse-Spin Asymmetries in Drell-Yan Processes, Eur. Phys. J. C 74 (2014) 2769 [arXiv:1307.5178] [INSPIRE].

[19] H.G. Cao, J.P. Ma and H.Z. Sang, Single Transverse Spin Asymmetries at Parton Level, Commun. Theor. Phys. 53 (2010) 313 [arXiv:0901.2966] [INSPIRE].

[20] J.P. Ma and H.Z. Sang, Soft-Gluon-Pole Contribution in Single Transverse-Spin Asymmetries of Drell-Yan Processes, JHEP 04 (2011) 062 [arXiv:1102.2679] [INSPIRE].

[21] J.P. Ma and Q. Wang, Scale Dependence of Twist-3 quark-gluon Operators for Single Spin Asymmetries, Phys. Lett. B 715 (2012) 157 [arXiv:1205.0611] [INSPIRE].

[22] J.C. Collins and D.E. Soper, Parton Distribution and Decay Functions, Nucl. Phys. B 194 (1982) 445 [inSPIRE].

[23] X.-D. Ji and J. Osborne, An analysis of the next-to-leading order corrections to the $g_{T}\left(=g_{1}+g_{2}\right)$ scaling function, Nucl. Phys. B 608 (2001) 235 [hep-ph/0102026] [INSPIRE].

[24] S. Meissner and A. Metz, Partonic pole matrix elements for fragmentation, Phys. Rev. Lett. 102 (2009) 172003 [arXiv:0812.3783] [INSPIRE].

[25] D. Boer and P.J. Mulders, Time reversal odd distribution functions in leptoproduction, Phys. Rev. D 57 (1998) 5780 [hep-ph/9711485] [INSPIRE]. 
[26] J.P. Ma and G.P. Zhang, QCD Corrections of All Structure Functions in Transverse Momentum Dependent Factorization for Drell-Yan Processes, JHEP 02 (2014) 100 [arXiv: 1308.2044] [INSPIRE].

[27] J.-w. Qiu and G.F. Sterman, High twist effects in hadronic collisions, AIP Conf. Proc. 223 (1991) 249 [InSPIRE].

[28] W. Vogelsang and F. Yuan, Next-to-leading Order Calculation of the Single Transverse Spin Asymmetry in the Drell-Yan Process, Phys. Rev. D 79 (2009) 094010 [arXiv:0904.0410] [INSPIRE].

[29] Z.-B. Kang, I. Vitev and H. Xing, Transverse momentum-weighted Sivers asymmetry in semi-inclusive deep inelastic scattering at next-to-leading order, Phys. Rev. D 87 (2013) 034024 [arXiv: 1212.1221] [INSPIRE]. 